\title{
Avaliação de intervenções de Gestão da Clínica na qualificação do cuidado e na oferta de leitos em um hospital público de grande porte
}

\author{
Evaluation of clinical governance interventions on qualification of care and \\ supply of beds in a large public hospital
}

\author{
Fernando Anschau ${ }^{1,2,3} \bowtie$, Jacqueline Webster ${ }^{1}$, Nelson Roessler ${ }^{1}$, Eduardo de Oliveira Fernandes ${ }^{1}$, \\ Viviane Klafke ${ }^{1}$, Carine Paim da Silva ${ }^{1}$, Gabriel Mersseshmidt ${ }^{1}$, Samantha Ferreira ${ }^{1}$, Sandra Maria Sales Fagundes ${ }^{1}$, \\ José Accioly Jobim Fossari ${ }^{1}$
}

${ }^{1}$ Hospital Nossa Senhora da Conceição. Porto Alegre, RS

${ }^{2}$ Centro de Educação Tecnológica e de Pesquisa em Saúde - Escola GHC, Grupo Hospitalar Conceição. Porto Alegre, RS.

${ }^{3}$ Escola de Medicina, Pontifícia Universidade Católica do Rio Grande do Sul (PUCRS). Porto Alegre, RS.

\section{RESUMO}

OBJETIVOS: Descrever os resultados alcançados em indicadores de desempenho hospitalar e na oferta de leitos com a estratégia de incorporação da Gestão da Clínica no processo assistencial da unidade de retaguarda do Hospital Nossa Senhora da Conceição.

MÉTODOS: O estudo foi realizado na unidade de retaguarda do Hospital Nossa Senhora da Conceição, em Porto Alegre, Rio Grande do Sul. A unidade de retaguarda é um setor de internação de curta permanência (abaixo de 10 dias de internação) destinado a pacientes do setor de emergência do hospital. No período do estudo essa unidade possuía capacidade para 27 leitos. Foram adotadas como ferramentas da Gestão da Clínica a implantação de equipes de referência multiprofissionais e de rounds multidisciplinares, a instituição do sistema Kanban para monitoramento do tempo médio de permanência; a introdução do Projeto Terapêutico Singular por ocasião do ingresso do paciente no hospital (setor de emergência); e a regulação interna dos leitos pelo Núcleo Interno de Regulação. Os indicadores hospitalares número de internações, tempo médio de permanência, resolubilidade, taxa de mortalidade e índice de rotatividade foram comparados entre os anos 2015 (antes da implementação da estratégia Gestão da Clínica) e 2016 (após a implementação da estratégia).

RESULTADOS: Após a introdução da Gestão da Clínica, houve aumento no número de internações de 1395 para 1537 ao ano. Ocorreram 1240 altas para o domicílio (média de 104 ao mês), mostrando um aumento de 101,9\% em relação ao período anterior. Também foi observada diminuição no número de transferências internas (entre a unidade de retaguarda e outros setores do hospital), aumento no índice de rotatividade de 51,6 para 56,9, diminuição no tempo médio de permanência de 7,2 dias para 6,6 dias e diminuição na taxa de mortalidade de 3,5 para 0,7 ( $\mathrm{p}<0.05$ ). CONCLUSÕES: A implantação da Gestão da Clínica no contexto do trabalho assistencial na unidade dos leitos de retaguarda do hospital em estudo associou-se a melhorias nos processos de cuidado, proporcionando maior oferta de leitos aos usuários.

DESCRITORES: gestão da clínica; governança clínica; administração de serviços de saúde; equipe interdisciplinar de saúde; relações interprofissionais; gestão da qualidade em saúde; sistema Kanban.

\section{ABSTRACT}

AIMS: To describe the results achieved in hospital performance indicators and supply of beds, with the strategy of incorporating clinical management into the care process of the backup unit of the Nossa Senhora da Conceição Hospital.

METHODS: The study was carried out in the backup unit of the Nossa Senhora da Conceição Hospital, in Porto Alegre, Rio Grande do Sul, Brazil. The backup unit is an inpatient hospital with beds intended for hospital emergency patients characterized by short stay (less than 10 days of hospitalization) and in the study period it had 27 beds. As clinic management tools we implemented multidisciplinary reference teams and multidisciplinary rounds, established a Kanban system to monitor mean length of stay, and introduced the unique therapeutic project at the hospital entrance (emergency room) and management of beds by the Internal Regulation Center. We monitored the hospital indicators number of hospitalizations, mean length of stay, resolvability, mortality rate and turnover rate over a period of 12 months (2016, after implementation of the strategy) and made comparisons with the same period of the previous year.

RESULTS: After the introduction of Clinic Management, there was an increase in the number of hospitalizations from 1395 to $1537 /$ year. There were 1240 discharges to home (an average of $104 \mathrm{a}$ month), showing an increase of $101.9 \%$ in relation to the previous period. There was also a decrease in the number of internal transfers (between the back unit and other sectors of the hospital), an increase in the turnover rate from 51.6 to 56.9 , decrease in the mean stay time of 7.2 days to 6.6 days and a significant decrease in the mortality rate from 3.5 to 0.7 ( $p<0.05$ ). CONCLUSIONS: The implementation of clinical management in the context of care work in the hospital's backup bed unit fostered improvements in care processes, as well as ensuring greater supply of beds to users.

KEY WORDS: clinical management; clinical governance; health services management; interdisciplinary health team; interprofessional relations; health quality management; Kanban System. 
Abreviaturas: HNSC, Hospital Nossa Senhora da Conceição; PTS, projeto terapêutico singular; TMP, tempo médio de permanência.

\section{INTRODUÇÃO}

A Gestão da Clínica pode ser entendida como práticas assistenciais e gerenciais desenvolvidas a partir da caracterização do perfil dos usuários por meio da gestão de leitos, co-responsabilização das equipes e avaliação de indicadores assistenciais [1]. Outro autor caracteriza a Gestão da Clínica como o conjunto de tecnologias de microgestão da clínica que tem por objetivo a provisão de uma atenção à saúde de qualidade, centrada nas pessoas, efetiva e segura, além de eficiente, oportuna, equitativa e ofertada de forma humanizada [2]. Algumas ferramentas da Gestão da Clínica já foram apontadas como estratégias que promovem a interação entre os diversos profissionais de saúde, em especial entre médicos e enfermeiros, qualificando o cuidado [3, 4].

Podemos destacar algumas ações com potencial para gerar resultados positivos na qualificação assistencial, na garantia de mais acesso e na organização administrativa/assistencial de um hospital como: o sistema Kanban, o Projeto Terapêutico Singular (PTS), as equipes de referência multiprofissional e os rounds multidisciplinares [5-7].

O sistema Kanban, no presente contexto, é uma ferramenta que deixa transparente e de fácil acesso todos os tempos médios de permanência (TMP) e a permanência dos pacientes de cada serviço/unidade hospitalar, possibilitando a lembrança do indicador a todos os profissionais envolvidos com a assistência. Para isso, o Kanban consta de uma lista informatizada com todos os leitos e pacientes da unidade de internação em questão, com informações sobre diagnóstico, tempo de permanência e previsão de alta, além do elenco de pendências para a alta do paciente. Através dessa ferramenta é possível uma classificação em cores sobre o status da internação, da seguinte forma: verde para tempo de internação dentro do previsto como adequado, amarelo no dia anterior ao previsto para a alta, vermelho para os casos em que o tempo estimado da alta foi ultrapassado [5].

O PTS é um conjunto de propostas de condutas terapêuticas articuladas para um paciente, resultado da discussão coletiva de uma equipe interdisciplinar. $\mathrm{Na}$ abordagem do paciente no setor de emergência do hospital utilizam-se as avaliações médicas, da enfermagem, da fisioterapia, da nutrição e do serviço social para compor o PTS. O PTS possibilita um olhar multidisciplinar para iniciar o processo do cuidado a cada paciente admitido na unidade $[6,7]$.

As equipes de referência multiprofissionais são compostas por profissionais de diversas áreas, como médicos, farmacêuticos, enfermeiros, assistentes sociais, nutricionistas e fisioterapeutas, com objetivo de promover a qualidade da atenção ao usuário, a agilização dos processos de trabalho e a qualificação da alta hospitalar. Nos rounds multidisciplinares, as equipes de referência tratam os casos clínicos dos pacientes internados, as pendências relacionadas ao diagnóstico e terapêutica e a previsão para a alta hospitalar, agregando melhor as discussões e tornando mais clara a condução dos casos. O grupo de ferramentas de gestão na construção da Gestão da Clínica é variado e pode ser aplicado com diferentes intensidades, adaptando-se a cada realidade organizacional [6-8].

Ao propor mudanças na gestão do trabalho assistencial, é importante utilizar indicadores capazes de demonstrar os resultados após a intervenção. Os indicadores número de internações e índice de rotatividade podem demonstrar, quando em elevação, que está sendo proporcionada maior oferta de leitos pelo hospital. O menor tempo médio de permanência (TMP) dos pacientes, além de contribuir para a maior oferta de leitos, pode indicar maior capacidade resolutiva da equipe assistencial. $\mathrm{O}$ indicador taxa de mortalidade atua como forte balizador da qualidade assistencial, sendo identificada melhora na assistência quando seus resultados são menores.

O objetivo deste estudo foi descrever os resultados alcançados nos indicadores TMP, número de internações, taxa de mortalidade e índice de rotatividade, com a estratégia de incorporação da Gestão da Clínica no processo assistencial da unidade de retaguarda do Hospital Nossa Senhora da Conceição (HNSC).

\section{MÉTODOS}

O HNSC, um hospital público geral com 843 leitos e uma taxa de ocupação superior a $90 \%$, está localizado em Porto Alegre, capital do Rio Grande do Sul. O presente estudo foi realizado na unidade de retaguarda desse hospital. A unidade de retaguarda é um setor de internação de curta permanência (tempo de permanência hospitalar abaixo de 10 dias de internação) destinado a pacientes do setor de emergência. No período do estudo essa unidade possuía capacidade para 27 leitos, sob a responsabilidade da equipe médica 
da Medicina Interna. Para tanto, foram introduzidas as seguintes medidas: adoção de equipes de referência multiprofissionais e de rounds multidisciplinares; instituição do sistema Kanban para monitoramento do TMP e das pendências para resolução do caso de cada paciente; introdução do PTS no setor de emergência, onde ocorre o ingresso do paciente no hospital; e regulação interna dos leitos pelo Núcleo Interno de Regulação.

Diante das características nosológicas e do perfil social dos pacientes do HSNC, além da disposição de recursos humanos, optou-se por formar equipes compostas por médicos clínicos gerais, enfermeiros, assistentes sociais, fisioterapeutas, nutricionistas e farmacêuticos. Durante dois meses (novembro e dezembro de 2015) a concepção de equipe - aqui tratada como multiprofissional - foi trabalhada e supervisionada pela equipe gerencial do hospital (apoio institucional). Considera-se que a dinâmica do round multidisciplinar pode explicitar melhor as intervenções necessárias para um olhar mais amplo sobre o paciente e suas necessidades assistenciais. $\mathrm{O}$ round foi definido como a reunião, que ocorria duas vezes por semana, de monitoramento e organização clínica de cada paciente, onde os assuntos atinentes a cada área de conhecimento da equipe e necessários à condução do caso eram discutidos, visando qualificar a assistência e concluir o tratamento da doença que trouxe o paciente à internação.

O sistema Kanban (a exemplo do utilizado na estratégia de administração de produção em uma indústria, no qual cartões de sinalização controlam os fluxos de produção ou transporte), foi utilizado para controle visual do tempo de permanência e, de acordo com a previsão de alta, com o apontamento (explícito no painel de acompanhamento) das pendências para tanto [5]. A equipe responsável pela unidade dos leitos de retaguarda alimentava os dados sobre as pendências no Kanban, que estava ligado ao sistema informatizado do hospital, vinculado à prescrição. $\mathrm{O}$ sistema Kanban demonstrava, em forma de tabela, os leitos, nomes e idades dos pacientes, bem como seu tempo de permanência no hospital e as pendências para a efetivação das medidas clínicas na internação e para a alta hospitalar.

O PTS, como ferramenta multidisciplinar, foi a estratégia usada para qualificar a identificação dos diversos aspectos que deveriam ser abordados para um cuidado integral do paciente na internação e no plano de alta, com o envolvimento do próprio paciente e de familiares quando necessário. Em verdade, como citado na literatura, o PTS funciona como uma variação das reuniões para discussão de caso clínico, mas utilizada de forma sistemática para todos os pacientes $[6,7]$.

Após o período de dois meses (últimos dois meses de 2015) de introdução da tecnologia Gestão da Clínica, já com as concepções das ferramentas acima citadas incorporadas ao processo assistencial, foi iniciado o monitoramento dos resultados da estratégia para compor o presente estudo. Foi feito um fechamento preliminar dos dados após seis meses e, com resultados animadores na qualificação assistencial e em maior acesso hospitalar, os mesmos foram publicados [8]. A manutenção da estratégia e o monitoramento prolongado, durante o período de janeiro a dezembro de 2016, foi importante para observar o impacto da Gestão da Clínica no número de internações, TMP, taxa de mortalidade e índice de rotatividade da unidade de leitos de retaguarda do HNSC por um período mais estendido. Através do sistema informatizado do Grupo Hospitalar Conceição (GHC) efetuou-se a comparação dos resultados no período do estudo com aqueles do ano de 2015, quando a estratégia Gestão da Clínica não se fazia presente na unidade.

Os indicadores seguiram as seguintes definições: (i) como número de internações consideramos o número de pacientes admitidos para ocupar um leito hospitalar por um período igual ou maior a 24 horas; (ii) por TMP foi considerada a relação entre total de pacientes dia e o total de pacientes que tiveram saída do hospital em determinado período, incluindo óbito; (iii) o índice de rotatividade, que representa a utilização do leito hospitalar durante o período, considerado indicador hospitalar de produtividade, foi calculado como o número de saídas dividido pelo número de leitos no mesmo período; (iv) a taxa de mortalidade reflete a relação entre o número de óbitos ocorridos em pacientes internados e o número de pacientes que tiveram saída do hospital, em determinado período; (v) como resolubilidade do setor foi utilizado o número de altas para a residência no período, aqui classificadas como "com resolução adequada" enquanto o número de transferências internas para outros setores do hospital e/ou o óbito do paciente foram classificados como "sem resolução adequada" [9-11].

A distribuição dos dados foi apresentada em frequência e percentuais e o teste de Qui-quadrado foi usado para avaliar a associação entre a Gestão da Clínica e as taxas de mortalidade e de alta hospitalar. Os valores de $\mathrm{p}$ inferiores a 0,05 foram considerados significativos. A análise estatística foi realizada utilizando o programa SPSS Statistics versão 17.

$\mathrm{O}$ estudo foi aprovado e recomendado para publicação pela Direção do HNSC (documento HNSCGUI-012/17). 


\section{RESULTADOS}

Durante o ano de 2015, antes da implantação da estratégia Gestão da Clínica, o número de internações na unidade de retaguarda foi de 1395, (média de 116 internações por mês). O número de transferências internas nesse período de 12 meses foi de 726 pacientes. Ocorreram 619 altas hospitalares (média de 51,5 ao mês). Em 2016, após a introdução da Gestão da Clínica, o número de internações aumentou para 1537 (média de 128 internações ao mês), um acréscimo de 142 internações no ano, para o mesmo número de leitos. Ocorreram 1240 altas (média de 104 ao mês), mostrando um aumento de $101,9 \%$ em relação ao período anterior. O número de transferências internas diminuiu, e o índice de rotatividade aumentou de 51,6 para 56,9 (Tabela 1).

No período de janeiro a dezembro de 2015 o TMP foi de 7,2 dias enquanto que no ano de 2016, após a implantação da estratégia Gestão da Clínica, o TMP foi de 6,6 dias, ainda com potencial para diminuição, uma vez que em abril de 2016 o mesmo foi de 5,8 dias. A taxa de mortalidade diminuiu de 3,5 para 0,7 na unidade de retaguarda, comparando o ano de 2015 com o ano de $2016(p<0.05)$ (Tabela 1).

Tabela 1. Indicadores hospitalares antes e após a implantação da Gestão da Clínica na unidade de retaguarda (27 leitos) do Hospital Nossa Senhora da Conceição em Porto Alegre, RS, nos anos de 2015 e 2016.

\begin{tabular}{lcc} 
& \multicolumn{2}{c}{ Período } \\
\cline { 2 - 3 } \multicolumn{1}{c}{ Indicador } & $\begin{array}{c}\mathbf{2 0 1 5} \\
\text { (sem Gestão } \\
\text { da Clínica) }\end{array}$ & $\begin{array}{c}\mathbf{2 0 1 6} \\
\text { (com Gestão } \\
\text { da Clínica) }\end{array}$ \\
\hline Internações (n) & $\mathbf{1 . 3 9 4}$ & $\mathbf{1 . 5 3 7}$ \\
Transferências & 726 & 278 \\
Altas & 619 & 1.248 \\
Óbitos & 49 & 11 \\
Tempo médio de permanência (dias) & 7,2 & 6,6 \\
Taxa de mortalidade* (\%) & 3,5 & 0,7 \\
Índice de rotatividade (pacientes/leito) & 51,6 & 56,9 \\
\hline
\end{tabular}

* Qui quadrado: $\mathrm{p}<0.05$.

Tabela 2. Gestão da clínica e resolubilidade na unidade de retaguarda (27 leitos) do Hospital Nossa Senhora da Conceição em Porto Alegre, RS, nos anos de 2015 e 2016.

\begin{tabular}{lcccc}
\hline & $\begin{array}{c}\text { Com resolução } \\
\text { adequada* }^{*}\end{array}$ & $\begin{array}{c}\text { Sem resolução } \\
\text { adequada }^{+}\end{array}$ & Total & p $^{\ddagger}$ \\
\hline Com Gestão da Clínica & $1248(81,2 \%)$ & $289(18 \%)$ & $1537(100 \%)$ & $<0,05$ \\
Sem Gestão da Clínica & $619(44,4 \%)$ & $775(55,6 \%)$ & $1394(100 \%)$ & \\
\hline Total & $1867(63,6 \%)$ & $1064(36,4 \%)$ & $2931(100 \%)$ & \\
\hline
\end{tabular}

* Resolução adequada: alta hospitalar; + Sem resolução adequada: transferência para outra unidade ou óbito; ₹ Qui-quadrado.
Comparando os dois períodos (2015 com 2016) não houve mudança no perfil nosológico das internações e manteve-se a mesma distribuição etária e por sexo na unidade em estudo.

Analisando a resolubilidade da unidade hospitalar, considerada como capacidade de resolução clínica e alta hospitalar, sem transferência do cuidado a outras unidades ou óbito do paciente, foi possível observar maior potencial de resolução após a implantação da Gestão da Clínica. Verificou-se associação estatisticamente significativa entre a aplicação da Gestão da Clínica e a resolução da internação hospitalar $(\mathrm{p}<0.05)$ (Tabela 2).

\section{DISCUSSÃO}

Assim como já havia sido indicado em um estudo preliminar, após a implantação da Gestão da Clínica aumentou a atividade hospitalar por aumento da eficiência, medida tanto pelo TMP quanto pela taxa de mortalidade e pelo número de altas hospitalares. O período de um ano foi conclusivo para consolidar a estratégia e ratificar os resultados de qualificação assistencial e garantia de acesso apontados em nosso estudo anterior, com seis meses de desenvolvimento da Gestão da Clínica [8].

Quando buscamos o desenvolvimento do princípio da integralidade na assistência à saúde no ambiente hospitalar, necessariamente o fazemos através das equipes de saúde, pela sua compreensão das necessidades de saúde dos pacientes e pelo seu entendimento das estratégias do modo de atender àquelas necessidades [12]. A introdução de ferramentas de gestão objetivando implantar a Gestão da Clínica na unidade de retaguarda do HNSC proporcionou maior oferta de leitos aos pacientes e indicou maior qualificação assistencial, com aumento das altas hospitalares e diminuição das transferências internas e da taxa de mortalidade. No setor foram alocados recursos humanos que já dispunham de trabalho em equipes horizontais, o que facilitou a implantação da Gestão da Clínica. Entendemos que foi possível ampliar a concepção do papel do hospital para além da promoção, prevenção e assistência aos pacientes, incorporando a necessidade de gerenciamento e foco no usuário $[13,14]$.

No processo de organização do grupo de profissionais para a introdução das ferramentas da Gestão da Clínica, que incluiu o PTS, o sistema Kanban, as equipes de referência multiprofissionais e os rounds 
multidisciplinares, a presença de um Núcleo Interno de Regulação foi de extrema importância, uma vez que os pacientes classificados com o perfil nosológico para os leitos de retaguarda puderam ser direcionados para a unidade com eficiência. O Núcleo Interno de Regulação possui o mapa de leitos hospitalares e a identificação dos pacientes que entram pela emergência do hospital, trabalhando ininterruptamente para a gestão de leitos. Essas equipes, trabalhando em conjunto e utilizando as ferramentas da Gestão da Clínica, possibilitaram a redução do TMP e o aumento aproximado de 10,2\% no número de internações, com elevação do índice de rotatividade. A menor duração das internações tem o potencial de diminuir a ocorrência de infecções hospitalares, aumentar o acesso da população ao sistema de saúde e reduzir os gastos assistenciais [15].

Já no momento da entrada no hospital, no setor de emergência, a adoção de uma ferramenta para registo do PTS foi de grande relevância para a identificação de ações facilitadoras da alta hospitalar, como por exemplo dificuldades sociais (pacientes sem residência fixa ou com desagregação familiar). Como explicam Campos e Amaral [16], o PTS consiste em um atendimento interdisciplinar, que inclui a contribuição de várias especialidades e profissões. A partir de uma avaliação compartilhada, são planejados os procedimentos que ficarão a cargo de cada membro da equipe multiprofissional, denominada equipe de referência. Cada profissional da equipe de referência tem o encargo de acompanhar os pacientes ao longo da internação, solicitando a intervenção de outros profissionais ou serviços de apoio quando necessário e, finalmente, proporcionando a alta e a continuação do acompanhamento em outro nível do sistema de saúde $[16,17]$. Essas medidas de responsabilização do cuidado tem demonstrado melhora na qualificação e segurança da alta hospitalar [18].

O PTS pôde classificar adequadamente os pacientes e a introdução dessa ferramenta proporcionou maior acurácia na regulação interna e direcionamento aos leitos, aqui caracterizados como de retaguarda ao setor de emergência. Classificar adequadamente não somente o paciente quanto a sua gravidade na porta de entrada do hospital (setor de emergência) mas também de acordo com as suas necessidades assistenciais é de grande relevância para o planejamento terapêutico e necessita de um claro entendimento dos profissionais sobre essa classificação [19]. O setor de emergência do HNSC já possui experiência em sistema de classificação de risco (a classificação de Manchester está implantada no setor) e as equipes de internação já classificam a gravidade dos pacientes pelo índice de comorbidades de Charlson, o que facilitou a introdução de sistema que visa a classificação e ordenamento interno dos pacientes [20-23]. A estratégia de aplicação do PTS na entrada dos pacientes tornou possível visualizar a integração dos processos a partir do usuário, entendendo como eles deveriam ser realizados ou modificados, para otimizar a internação hospitalar e qualificar a assistência [24].

Pela atuação da equipe multiprofissional, na visão de diferentes profissões e suas variadas abordagens ao processo da doença e do cuidado, podemos ampliar o espaço subjetivo do paciente, contribuindo para sua humanização [25]. A equipe multiprofissional e o funcionamento em rounds multidisciplinares de fato ampliaram o olhar aos pacientes e qualificaram a assistência na unidade de retaguarda, em especial quando observamos a diminuição das taxas de mortalidade e o aumento no número de altas hospitalares no período. A taxa de mortalidade caiu em mais de $100 \%$ e o número de altas entre os dois períodos do estudo dobrou. A diminuição de óbitos é fato de extrema relevância clínica, assim como o aumento no número de altas hospitalares. Considerando a persistência do perfil nosológico, etário e de distribuição por sexo dos pacientes da unidade estudada, a diminuição da taxa de mortalidade indica uma melhora significativa na resolubilidade e na qualidade da assistência entre os dois períodos.

Segundo Da Silva et al. [26] a efetivação do PTS nas equipes de saúde precisa incluir boa comunicação e integração entre a equipe, além da existência de espaços para discussão multidisciplinar. Assim sendo, enfatiza-se a construção do PTS como atividade rotineira a ser desenvolvida nos serviços de saúde. $\mathrm{O}$ PTS busca atender a demandas de saúde complexas e por isso a equipe multidisciplinar, deve adquirir e trocar conhecimentos, possibilitando também autonomia ao usuário, tornando-o sujeito ativo na construção do processo de saúde e priorizando a qualidade do cuidado [26]. Diversas intervenções que qualificam o processo de alta já foram apontadas na literatura, e a Gestão da Clínica, como abordada neste estudo, com seu pacote de ferramentas, contribui nessa direção, promovendo a cultura de uma alta hospitalar segura e qualificando os processos de comunicação entre as equipes [14]. A Gestão da Clínica aproxima áreas técnicas assistenciais às áreas estratégico-gerenciais, fortalecendo dessa maneira o próprio processo de gestão que busca a qualidade institucional [13].

Este estudo demonstra que a Gestão da Clínica pode aumentar o número de internações diminuindo o TMP, além de garantir maior resolução aos casos atendidos nos leitos da unidade de retaguarda, aumentando as 
altas hospitalares e diminuindo a taxa de mortalidade. Dessa forma, podemos concluir que a implantação da Gestão da Clínica no contexto do trabalho assistencial na unidade dos leitos de retaguarda do HNSC associou-se a melhorias nos processos de cuidado, proporcionando maior oferta de leitos aos usuários.

\section{NOTAS}

Declaração de conflito de interesses

Os autores declaram não haver conflitos de interesses relevantes ao conteúdo deste estudo, informam ter tido acesso a todos os dados obtidos e assumem completa responsabilidade pela integridade dos resultados.

\section{REFERÊNCIAS}

1. Brasil. Ministério da Saúde. Portaria n. 3.390, de 30 de dezembro de 2013. Institui a Política Nacional de Atenção Hospitalar no âmbito do Sistema Único de Saúde, estabelecendo-se as diretrizes para a organização do componente hospitalar da Rede de Atenção à Saúde. Diário Oficial da República Federativa do Brasil [Internet]. Brasília; 2013 [cited 2016 Dec 16]. Available from: http://bvsms.saude. gov.br/bvs/saudelegis/gm/2013/prt3390_30_12_2013.html

2. Mendes EV. As Redes de Atenção à Saúde. Brasília: Organização Pan-Americana da Saúde; 2011.

3. Tang CJ, Chan SW, Zhou WT, Liaw SY. Collaboration between hospital physicians and nurses: an integrated literature review. Int Nurs Rev. 2013;60(3):291-302. https://doi.org/10.1111/inr.12034

4. Gonzalo JD, Kuperman E, Lehman E, Haidet P. Bedside interprofessional rounds: perceptions of benefits and barriers by internal medicine nursing staff, attending physicians, and housestaff physicians. J Hosp Med. 2014;9(10):646-51. https://doi.org/10.1002/ jhm. 2245

5. Lopes dos Reis R. Manual da gestão de Stocks: teoria e prática. Lisboa: Editorial Presença; 2008.

6. Brasil. Ministério da Saúde. Secretaria de Atenção à Saúde. Núcleo Técnico da Política Nacional de Humanização. Clínica ampliada, Equipe de referência e projeto terapêutico singular. 2aㅡ. ed. Brasília: Ministério da Saúde; 2007. (Série B. Textos Básicos de Saúde).

7. Rea CM, Thompson GE. Multidisciplinary patient management by means of a high social risk screening tool. Clin Perform Qual Health Care. 1996;4(3):159-63.

8. Anschau F, Webster J, Roessler N, de Oliveira Fernandes, Klafke V, Paim da Silva C, Messerschmit G, Ferreira S, Fossari JAJ. Clinic Management as a Tool for Enhancing quality of Care and Guarantee of Hospital Access. BAOJ Med Nursing. 2016;2(5):1-4.

9. Zucchi P, Bittar OJN, Haddad N. Produtividade em hospitais de ensino no estado de São Paulo, Brasil. Rev Panam Salud Publica. 1998;4(5):311-6. https://doi.org/10.1590/S1020-49891998001100004

10. Travassos C, Noronha J, Martins M. Mortalidade hospitalar como indicador de qualidade: uma revisão. Ciênc Saúde Colet. 1999;4(2):367-81. https://doi.org/10.1590/S1413-81231999000200011

11. Machado JP, Martins ACM, Martins MS. Avaliação da qualidade do cuidado hospitalar no Brasil: uma revisão sistemática. Cad Saúde Pública. 2013;29(6):1063-82. https://doi.org/10.1590/S0102-311X2013000600004

12. Barros JO. A construção de projetos terapêuticos no campo da saúde mental: apontamentos acerca das novas tecnologias de cuidado [dissertação]. [São Paulo]: Universidade de São Paulo; 2010. 111 p.

13. Bonato VL. Gestão de qualidade em saúde: melhorando assistência ao cliente. Mundo Saude. 2011;35(5):319-31.

14. Williams H, Edwards A, Hibbert P, Rees P, Prosser Evans H, Panesar S, Carter B, Parry G, Makeham M, Jones A, Avery A, Sheikh A, Donaldson L, Carson-Stevens A. Harms from discharge to primary care: mixed methods analysis of incident reports. Br J Gen Pract. 2015;65(641):e829-37. https://doi.org/10.3399/bjgp15X687877

15. Husser J, Guerin O, Bretones D. The incentive effects of DRG's reimbursement rates for health care estabilishments in France: to wards a new allocation of surgical procedures? Int Bus Research. 2012;5(12):31-7. https://doi.org/10.5539/ibr.v5n12p31

16. Campos GWS, Amaral MA. A clínica ampliada e compartilhada, a gestão democrática e redes de atenção como referenciais teóricooperacionais para a reforma do hospital. Ciênc Saúde Colet. 2007;12(4) :849-59. https://doi.org/10.1590/S1413-81232007000400007

17. Pinto DM, Jorge MSB, Pinto AGA, Vasconcelos MGF, Cavalcante CM, Flores AZT, de Andrade AS. Projeto terapêutico singular na produção do cuidado integral: uma construção coletiva. Texto Contexto Enferm. 2011;20(3):493-502. https://doi.org/10.1590/S010407072011000300010

18. Yu SY, Ko IS, Lee SM, Park YW, Lee C. A unit-coordinator system: an effective method of reducing inappropriate hospital stays. Int Nurs Rev. 2011;58(1):96-102. https://doi.org/10.1111/j.1466-7657.2010.00850.x

19. Sendlhofer G, Brunner G, Tax C, Falzberger G, Smolle J, Leitgeb K, Kober B, Kamolz LP. Systematic implementation of clinical risk management in a large university hospital: the impact of risk managers. Wien Klin Wochenschr. 2015 Jan;127(1-2):1-11. https://doi. org/10.1007/s00508-014-0620-7

20. Martins M, Blais R, de Miranda NN. Avaliação do índice de comorbidade de Charlson em internações da região de Ribeirão Preto, São Paulo, Brasil. Cad Saude Publica. 2008 Mar;24(3):643-52. https://doi.org/10.1590/S0102-311X2008000300018

21. Charlson ME, Pompei P, Ales KL, MacKenzie CR. A new method of classifying prognostic comorbidity in longitudinal studies: development and validation. J Chronic Dis. 1987;40(5):373-83. https://doi.org/10.1016/0021-9681(87)90171-8 
22. Martins HM, Cu-a LM, Freitas P. Is Manchester (MTS) more than a triage system? A study of its association with mortality and admission to a large Portuguese hospital. Emerg Med J. 2009 Mar;26(3):183-6. https://doi.org/10.1136/emj.2008.060780

23. Rafael MS, Portela SL, Sousa P, Fernandes AC. Utilização do serviço de urgência pediátrica: a experiência de um centro português. Sci Med. 2017;27(1):ID24919. https://doi.org/10.15448/1980-6108.2017.1.24919

24. Lopes de Figueiredo M, D’Innocenzo M. Dificuldades encontradas pelos gestores de instituição de saúde na utilização da metodologia gestão por processos. RAS. 2011;13(50):45-52

25. Fernandes JCL. A quem interessa a relação médico paciente? Cad Saúde Pública. 1993;9(1):21-7. https://doi.org/10.1590/S0102$311 X 1993000100003$

26. Da Silva EP, Pereira de Melo FA, Marques de Souza M, Gouvea RA, Andrade e Tenório A, Cabral AFF, Pacheco MCS, Andrade AFR, Pereira TM. Projeto terapêutico singular como estratégia de prática da multiprofissionalidade nas ações de saúde. R Bras Ci Saúde 2013;17(2):197-202. https://doi.org/10.4034/RBCS.2013.17.02.14 